CASE REPORT

\title{
Pneumocystis carinii pneumonia in chronic lymphocytic leukaemia
}

\author{
S R Vavricka, J Halter, L Hechelhammer, A Himmelmann
}

Postgrad Med J 2004;80:236-238. doi: 10.1136/pgmi.2003.012252

Pneumocystis carinii pneumonia in patients with chronic lymphocytic leukaemia (CLL) who have not been treated with fludarabin are rare, although clinically relevant CD4 T-cell depletion can occur in longstanding CLL without prior treatment with purine analogues. A 52 year old woman is reported who was on long term treatment with chlorambucil and taking a short course of prednisone for familial CLL before she developped progressive dyspnoea, and $P$ carinii pneumonia was diagnosed in bronchoalveolar lavage fluid. Despite treatment with high dose co-trimoxazole the patient died.

nfections are a major cause of morbidity and mortality in patients with chronic lymphocytic leukaemia (CLL). Predisposition to infection in CLL is mediated through various abnormalities including both the impairment of humoral and cellular immunity and further immunosuppression related to the therapy of CLL. ${ }^{1}$ Among the infections in CLL patients, pulmonary manifestations are very common and are often difficult to distinguish from other pulmonary disorders on clinical grounds. Opportunistic infections such as Pneumocystis carinii, fungi, viruses, and mycobacteria are seen in patients with CLL, most commonly in those being treated with corticosteroids and/or purine analogues. ${ }^{2}{ }^{3} P$ carinii pneumonia in CLL patients who have not been treated with fludarabin, however, is rare.

We describe a patient with familial CLL and a very low CD4 count who developed a $P$ carinii pneumonia infection in the absence of fludarabine therapy.

\section{CASE REPORT}

A 52 year old woman with a 10 year history of familial CLL was treated with chlorambucil (pulsed treatment with chlorambucil at a daily dose of $10 \mathrm{mg}$ for three consecutive days every four to eight weeks). This regimen led to reversion of her anaemia. One of her brothers also had CLL as had her father, who died after a transformation of CLL into acute leukaemia. No other familial diseases were known and there was no family history of increased susceptibility to infectious diseases. Because of the development of autoimmune haemolytic anaemia (Coombs IgG, raised lactate dehydrogenase, reticulocytosis, decrease in haemoglobin concentration from $120 \mathrm{~g} / \mathrm{l}$ to $90 \mathrm{~g} / \mathrm{l}$ ) five weeks before admission to our hospital, steroid therapy of $50 \mathrm{mg}$ prednisolone for one week was given with consecutive tapering of the steroid dose and improvement of the haemoglobin value.

She was admitted to an external hospital because of fatigue, prolonged productive cough, progressive dyspnoea, and a weight loss of $12 \mathrm{~kg}$. Additional history was significant with herpes zoster infection of the right forearm, which was treated with valaciclovir, clomipramin, carbamazepin, and haloperidol four weeks before admission. Chest radiography revealed a discrete confluent infiltration of the left lower lobe; the lactate dehydrogenase on admission was $1561 \mathrm{U} / \mathrm{l}$. Treatment with clarithromycin was initiated. Because the condition of the patient deteriorated, she was transferred to our hospital. Physical examination on admission showed an ill appearing white woman. Her temperature was $37.4^{\circ} \mathrm{C}$. There was no adenopathy and the spleen was not palpable. Chest radiography (fig lA) and computed tomography scan of her thorax showed diffuse interstitial infiltration. We changed the antibiotics to high dose co-trimoxazole therapy. The white cell count on admission was $25.6 \times 10^{9} / 1(53 \%$ lymphocytes and $16.5 \%$ smudge cells). An anaemia of $7.5 \mathrm{~g} / \mathrm{l}$ and a thrombopenia of $36 \times 10^{9} / \mathrm{l}$ had developed. No decreased serum IgM, IgG, and IgA levels were found. After respiratory decompensation she was intubated and a bronchoscopy with bronchoalveolar lavage was performed. Immunofluorescence and Giemsa staining revealed the presence of $P$ carinii. Furthermore herpes simplex was found by viral culture and intravenous aciclovir was added to the therapy with high dose co-trimoxazole. The patient had a low CD4 count of 104/ $\mu \mathrm{l}$ and a CD4/CD8 ratio of 0.4 . Serology for HIV, urinary legionella antigen detection, and cytomegalovirus were negative as well as cultures of blood, urine, stool, and cerebrospinal fluid. Bone marrow aspiration and a biopsy specimen showed a hypercellular bone marrow with decreased megakaryopoiesis and reduced erythropoiesis together with an infiltration of $60 \%$ of mature appearing lymphocytes. Immunophenotypic analysis of peripheral blood lymphocytes showed a monoclonal lymphocytic population expressing for CD5, CD23, and CD19. The patient eventually died in multiorgan failure.

Necropsy findings included leukaemic infiltrations in the spleen, lymph nodes, and the bone marrow. Examination of the lungs revealed abundant intra-alveolar $P$ carinii organisms (fig $1 \mathrm{~B}$ and $\mathrm{IC}$ ) and a severe diffuse alveolar damage (fig 1D) with intra-alveolar calcifications. ${ }^{4}$

Furthermore a blastic, angiocentric, Epstein-Barr virus positive (in situ hybridisation with DAKO EBER PNA Probe) $\mathrm{B}$-cell infiltration of the colon with ulceration and perforation was found (fig $2 \mathrm{~A}-\mathrm{C}$ ).

\section{DISCUSSION}

We report a patient in whom clinically relevant CD4 T-cell depletion had occurred in longstanding CLL without prior treatment with purine analogues. In addition $P$ carinii infection, herpes simplex virus reactivation, and development of a blastic, angiocentric, Epstein-Barr virus positive B-cell lymphoproliferation were found.

$P$ carinii is well known to cause opportunistic pulmonary infections, especially in immunodeficient patients infected with HIV. Although the method of transmission of $P$ carinii is not clearly understood, reactivation of latent infection due to immunosuppression may be one important mechanism of the disease. $P$ carinii pneumonia is a known complication in CLL patients treated with fludarabine and steroids. ${ }^{2}$ In CLL 


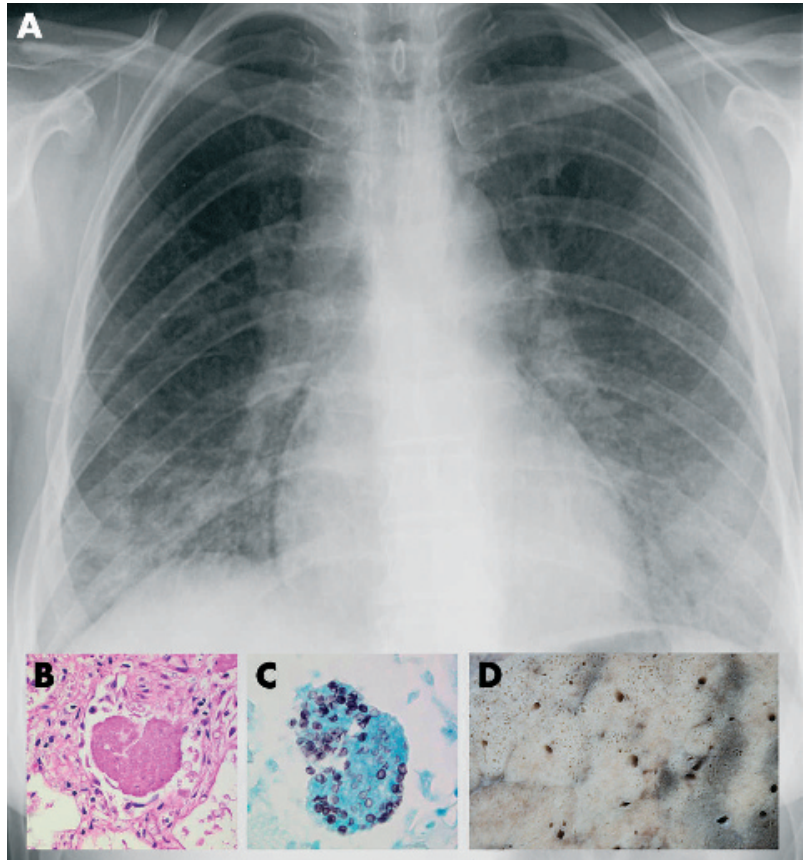

Figure 1 Chest radiograph $(\mathrm{A})$ showing confluent infiltrations of the left and right lower lobe. Macrosopic detail of the left upper lobe showing a loss and diffuse consolidation of the lung structure (D). Haematoxylin and eosin (B) and Grocott (C) stains on this lung specimen showing

Pneumocystis carinii organisms (magnification $\times 250$ ).

patients not treated with purine analogues $P$ carinii pneumonia is usually associated with steroid therapy over a long period of time, often in combination with cyclophosphamide. $^{35-8}$ In one large series the occurrence of $P$ carinii pneumonia in four patients with CLL is described but no clinical details regarding treatment or CD4 counts are provided. $^{3}$

Hypogammaglobulinaemia is probably the most important immune defect in terms of risk of severe bacterial infections in CLL patients. In patients with CLL, hypogammaglobulinaemia shows a prevalence from $10 \%$ to $100 \%$ and is correlated with the duration of the disease and with the stage of CLL. However in the patient presented here several immunoglobulin measurements were within the normal range.

A T-cell defect has also been described in CLL patients. In many patients with CLL the number of CD4 cells is actually increased but decreases as the disease progresses. More consistently, the CD4/CD8 ratio appears to decrease with advanced CLL, but such a low ratio as seen in our patient has never been reported. ${ }^{9}{ }^{10}$ In all CLL patients suffering from $P$ carinii pneumonia not treated with purine analogues, the number of CD4 cells was reported to be normal or only slightly reduced (range 300-370). ${ }^{6}$ Also the CD4/CD8 ratio was only slightly reduced. In contrast, in our patient the number of CD4 cells and the CD4/CD8 ratio were severely depressed. We could only find one other patient suffering from CLL with such low CD4 numbers; this patient developed a mycobacterial infection. ${ }^{11}$ As in our patient this patient had longstanding disease that lasted approximately 10 years and was treated with chlorambucil and intermittently with prednisone. Therefore longstanding CLL might lead to depletion of CD4 T-cells and clinicians might therefore consider a prophylactic antibiotic in these patients.

Surprisingly the necropsy showed perforation of the colon caused by a blastic, angiocentric, Epstein-Barr virus positive B-cell infiltration. Uncontrolled sepsis originating from the
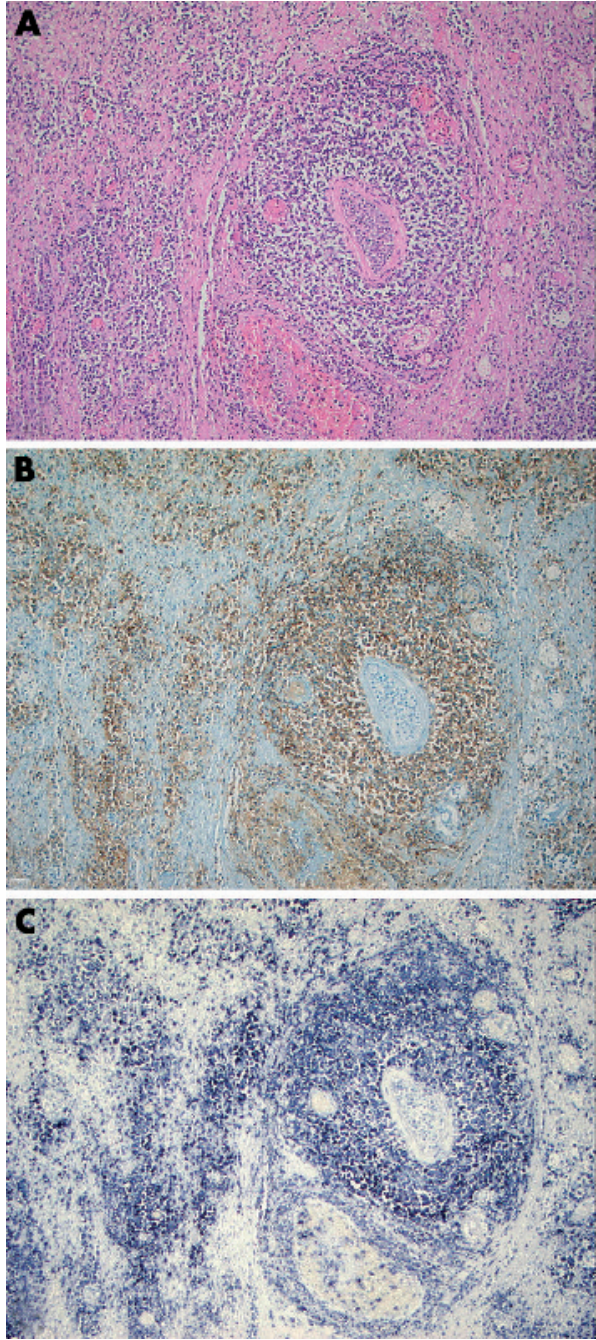

Figure 2 Blastic, angiocentric, Epstein-Barr virus positive B-cell infiltration from colon: haematoxylin and eosin stain (A), CD 20 immunostaining (B) and in situ hybridisation (DAKO EBER PNA Probe) for Epstein-Barr virus (C) (magnification $\times 65$ ).

perforation was probably the ultimate cause of death in this patient. The course of the disease in the present patient demonstrates that clinically relevant CD4 T-cell depletion can occur in longstanding CLL in the absence of prior treatment with purine analogues.

\section{Learning points}

- Opportunistic infections such as Pneumocystis carinii, fungi, viruses, and mycobacteria can occur in patients with chronic lymphocytic leukaemia (CLL) having treatment with long term corticosteroids and/or purine analogues.

- A clinically relevant CD4 T-cell depletion and/or a hypogammaglobulinaemia can occur in longstanding CLL without prior treatment with purine analogues.

- Therefore, patients with long lasting CLL without prior purine analogue treatment might be considered for a prophylaxis similar to those patients with CLL treated with purine analogues. 


\section{Authors' affiliations}

S R Vavricka, J Halter, A Himmelmann, Department of Medicine,

University Hospital of Zurich, Switzerland

L Hechelhammer, Department of Pathology, University Hospital of

Zurich, Switzerland

Correspondence to: Dr Andreas Himmelmann, Department of Medicine, University Hospital of Zurich, Raemistrasse 100, CH-8091

Zurich, Switzerland; andreas.himmelmann@dim.usz.ch

Submitted 15 July 2003

Accepted 8 October 2003

\section{REFERENCES}

1 Tsiodras S, Samonis G, Keating MJ, et al. Infection and immunity in chronic lymphocytic leukemia. Mayo Clin Proc 2000;75:1039-54.

2 Anaissie EJ, Kontoyiannis DP, O'Brien S, et al. Infections in patients with chronic lymphocytic leukemia treated with fludarabine. Ann Intern Med 1998;129:559-66.
3 Pagano L, Fianchi L, Mele L, et al. Pneumocystis carinii pneumonia in patients with malignant haematological diseases: 10 years' experience of infection in GIMEMA centres. Br J Haematol 2002; 1 17:379-86.

4 Lee M. Schinella R. Pulmonary calcification caused by Pneumocystis carinii

pneumonia. Am J Surg Pathol 1991;15:376-80.
5 Sen RP, Walsh TE, Fisher W, et al. Pulmonary complications of combination therapy with cyclophosphamide and prednisone. Chest 1991;99:143-6.

6 Taillan B, Garnier G, Ferrari E, et al. Leucémie lymphoide chronique et pneumocystose: trois observations. Ann Med Interne 1991;142:464-6.

7 Dworzack DL, Ferry JJ, Clark RB. Co infection with Legionella pneumophila and Pneumocystis carinii in a patient with chronic lymphocytic leukaemia. Nebr Med J 1989;74:73-5.

8 Peters SG, Prakash UB. Pneumocystis carinii pneumonia. Review of 53 cases. Am J Med 1987;82:73-8.

9 Hautekeete ML, De Bock RF, Van Bockstaele DR, et al. Flow cytometric analysis of T-lymphocyte subpopulations in B-cell chronic lymphocytic leukemia: correlation with clinical stage. Blut 1987; 55:447-52.

10 Totterman TH, Carlsson M, Simonsson B, et al. T-cell activation and subset patterns are altered in $\mathrm{B}-\mathrm{CLL}$ and correlate with the stage of the disease. Blood 1989;74:786-92.

11 Krebs T, Zimmerli S, Bodmer T, et al. Mycobacterium genavense infection in a patient with long-standing chronic lymphocytic leukaemia. J Intern Med 2000;248:343-8. 